\title{
Spinal Anesthesia with Low Doses of Hyperbaric Bupivacaine is Safe and Necessary in Brachytherapy for Carcinoma of the Cervix
}

\author{
Siddharta Lacerda ${ }^{1}$, Luiz Eduardo Imbelloni ${ }^{1, ~}{ }^{\text {, }}$, Maria das Graças Dantas Lima ${ }^{2}$ \\ ${ }^{1}$ Hospital Estadual of Trauma, João Pessoa, Brazil \\ ${ }^{2}$ Hospital of Câncer Napoleão Laureano, João Pessoa, Brazil \\ Email address: \\ siddharta_lacerda@hotmail.com (S. Lacerda), dr.imbelloni@hotmail.com (L. E. Imbelloni), mgdl@hotmail.com (M. das G. D. Lima) \\ ${ }^{*}$ Corresponding author
}

To cite this article:

Siddharta Lacerda, Luiz Eduardo Imbelloni, Maria das Graças Dantas Lima. Spinal Anesthesia with Low Doses of Hyperbaric Bupivacaine is Safe and Necessary in Brachytherapy for Carcinoma of the Cervix. American Journal of Applied Scientific Research.

Vol. 4, No. 1, 2018, pp. 1-4. doi: 10.11648/j.ajasr.20180401.11

Received: March 9, 2018; Accepted: March 26, 2018; Published: May 4, 2018

\begin{abstract}
Background and objectives: Cervical cancer occupies the third place among women. In advanced cases the treatment of choice is brachytherapy and pain is among the most frequent complaints of treatment. The objective of the study was to compare the incidence of pelvic pain and satisfaction among groups of patients submitted to brachytherapy with and without anesthesia. METHODS: Prospective, longitudinal study at a SUS hospital with 30 patients undergoing brachytherapy between the ages of 18 and 60 divided into three groups: GN (without anesthesia), GR5 (spinal anesthesia with $5 \mathrm{mg}$ of hyperbaric bupivacaine $0.5 \%$ ) and GR10 (spinal anesthesia with $10 \mathrm{mg}$ of $0.5 \%$ hyperbaric bupivacaine). Pain was evaluated immediately after brachytherapy (T1), 30 minutes (T2) and before discharge from the post-anesthetic recovery room (T3), through visual analogue scale and satisfaction with the technique. Effects of both doses administered. RESULTS: In the GN $70 \%, 50 \%$ and $10 \%$ felt pain, respectively, in T1, T2 and T3; in GR5 no patient presented pain in the 3 times studied; in GR 10 , only one patient had pain in T1. Regarding satisfaction, in GN $20 \%$ was poor, $60 \%$ satisfactory and $20 \%$ good; in GR5 $100 \%$ was good; in GR10, $60 \%$ was satisfactory, $40 \%$ good. CONCLUSION: Spinal anesthesia with low doses of hyperbaric bupivacaine is safe and necessary in BTCC. In conclusion, after this small study, spinal anesthesia became routine in this procedure and possibly to be implanted in all Brazilian Health System (SUS) units.
\end{abstract}

Keywords: Spinal Anesthesia, Hyperbaric Bupivacaine, Brachytherapy, Ambulatory

\section{Introduction}

Cervical cancer is the third most frequent tumor in the female population, behind breast and colorectal cancer, and the fourth leading cause of cancer death in Brazil [1]. For the year 2016, in Brazil, 16,340 new cases of cervical cancer are expected [2].

Brachytherapy, the implantation of radioactive sources in or near tumours, has acquired an important role in the treatment of a variety of cancers, and this modality uses radioactive sources through applicators in close contact with the region to be treated [3]. Brachytherapy forms an important part of the curative treatment of carcinoma cervix and we use manual after loading low dose rate (LDR) brachytherapy in the form of intracavitory application or transperineal interstitial template application. Pain ranks third among the most frequent complaints of patients undergoing treatment for cervical cancer, and the painful sensation during the placement of the applicators is a frequent complaint [4].

There are various anesthetic techniques described for high dose rate, intracavitory radiotherapy for carcinoma cervix i.e., conscious sedation, local infiltration, regional anesthesia (spinal or epidural) and general anaesthesia. Spinal anesthesia is a good option for outpatient procedures, since it has a low postoperative morbidity, in addition to presenting high patient satisfaction [5]. In the Brazilian oncology reference service (Brazilian Health System - SUS) in which this work was performed, there is no 
routine regarding anesthesia in brachytherapy for carcinoma of the cervix (BTCC).

The objective of this study was to compare the incidence of pelvic pain and satisfaction among groups of patients without using any anesthetic technique with groups submitted to spinal anesthesia for BTCC. Therefore, a decision was made to conduct a prospective study that compared a relatively standard dose of hyperbaric bupivacaine $(10 \mathrm{mg})$, with a lower dose $(5 \mathrm{mg})$, for spinal anesthesia. The aim was to provide adequate anesthesia using the lower dose, as well as allowing for a shorter time to complete recovery. The study findings could then be used to contribute to a guideline for spinal anesthesia for BTCC in patients from the Brazilian Health System.

\section{Method}

After registration in the Brazil Platform (CAAE: 58392416.6.0000.5179), approval by the ethics committee on research and signing of the informed consent, a double-blind randomized prospective study was performed in patients ASA physical status I and II (American Society of Anesthesiology), aged between 18 and 60 years old, weighing between 50 and $80 \mathrm{~kg}$ of women indicated for BTCC, in the lithotomy position and on an outpatient basis, did part of this prospective, double blind, randomized study. Patients with heart or respiratory disease, mental disorder, neurological disease, sensitivity to anesthetic or anticoagulant therapy were excluded.

As the total number of monthly patients is 48 , submitted to this type of procedure. Using a significance level of 5\% and a margin of error of 0.10 , sample size obtained was 30 patients, and 10 were allocated in each group.

No premedication was administered in the room. After venous puncture with $20 \mathrm{G}$ or $18 \mathrm{G}$ catheter was started, infusion of Ringer's lactate solution began. Monitoring in the operating room consisted of continuous ECG using the CM5 lead, blood pressure by non-invasive method and pulse oximetry.

The patients were randomly divided into three groups: GN (without anesthesia), GN5 (spinal anesthesia with $5 \mathrm{mg}$ of hyperbaric bupivacaine $0.5 \%$, Cristália Chemicals and Pharmaceuticals Ltd.) and GN10 (spinal anesthesia with 10 mg of hyperbaric bupivacaine $0.5 \%$, Cristália Chemicals and Pharmaceuticals Ltd.). In the GN group (control), the patients were placed in the brachytherapy room and the procedure was performed without any anesthetic technique (routine of the Service until the accomplishment of this study). In the groups GN5 and GN10, after cleaning the skin with alcohol, a subarachnoid puncture was performed with the patient in the sitting position, via the median route between the L3-L4 apophysis using a $27 \mathrm{G}$ needle with Quincke tip (B. Braun, Melsungen AG). After the appearance of cerebrospinal fluid in the needle, $1 \mathrm{~mL}$ of $0.5 \%$ hyperbaric bupivacaine in GN5 and $2 \mathrm{~mL}$ of the same solution in GN10 were injected at a rate of $1 \mathrm{~mL} / 4$ seconds. Immediately after the patient was placed in the horizontal dorsal decubitus, and after the installation of the sensory block, placed in a lithotomy position.

The time for the blockade installation was evaluated by the loss of the thermal sensitivity to the touch with a cotton ball soaked in alcohol along the midaxillary line, lateral side of the thigh, leg and foot. The evaluation of the motor block was performed at 10, 60 and 120 minutes after the spinal block, using the modified Bromage chart ( 0 to 3$)$ : $0=$ free movement of the feet and lower limbs; $1=$ inability to lift the extended limb (flex the thigh and keep the leg extended); $2=$ inability to bend knees; 3 = inability to move ankle. The duration of the blockade was defined as the time between puncture and injection of the solution and the recovery of perineal sensitivity at the touch of the needle stylus on the buttocks, evaluated every 15 minutes during the first hour and 30 minutes after the second hour. The duration of the surgery was evaluated in all three groups. In groups where there was spinal anesthesia the surgical time was timed after release by the anesthetic team. At the end of the surgery, the patients' ability to move from the surgical table to the transport stretcher without assistance was evaluated.

The pain was evaluated in three moments, immediately after brachytherapy (T1), through visual analogue scale (VAS), at 30 minutes after (T2) and before discharge from the post-anesthetic recovery room (T3), by question of YES if there was pain or NO in his absence. In $\mathrm{T} 1$, analgesic rescue with $50 \mathrm{mg} / \mathrm{kg}$ dipyrone was used in patients with mild pain (VAS 1-3) and tramadol $1 \mathrm{mg} / \mathrm{kg}$ in patients with moderate pain (VAS 4-6) and intense pain (VAS 7-10).

Hypotension was defined as the reduction of systolic pressure greater than $30 \%$ of the baseline value, being treated with ephedrine $(10 \mathrm{mg})$. Bradycardia was defined as reducing heart rate below 50 beats per minute and being treated with atropine $0.5 \mathrm{mg}$ intravenously. The occurrence of nausea and vomiting was investigated immediately after the end of brachytherapy. All patients received oxygen $(2 \mathrm{~L} / \mathrm{min})$ through a nasal catheter. After surgery, patients were transferred to the post-anesthetic care unit (PACU) for continuous monitoring of vital signs until complete blockade regression. Before hospital discharge, a resident anesthetist verified the patient's satisfaction with the technique and requested its classification as good, satisfactory or bad. Discharge was only allowed for patients who were awake, able to walk unassisted, and had stable vital signs. The patients were evaluated by telephone in relation to the appearance of a transient neurological deficit and postdural puncture headache.

\section{Results}

We studied 30 patients for BTCC, and none were excluded from the study. All patients under age group of 25-60 years. The groups did not differ in demographic variables (Table 1).

The onset of analgesia was rapid and comparable between two groups studied. The dose employed had no influence at the beginning of the blockade, defined as latency time (Table 2). The dispersion of analgesia was different between the two groups (Table 2). The level the median test was lower in the group with $5 \mathrm{mg}$, compared to $10 \mathrm{mg}$. The low-dose group had a lower peak sensory dermatome level (T10 with $5 \mathrm{mg}$ 
and $\mathrm{T} 8$ with $10 \mathrm{mg}$ ) (Table 2). The $5 \mathrm{mg}$ increase in dose corresponded to a significant increase of two fashionable segments between two doses used (Table 2). The duration of procedure was in the range of 4-15 minutes, without significant difference, most of the sessions were completed in 10 minutes (Table 2). The duration of the blockade was significantly higher with the higher dose (Table 2). When doubling the dose of 5 to $10 \mathrm{mg}$ of hyperbaric bupivacaine there was practically double the increase in blocking time (Table 2). All patients in the group without anesthesia and the $5 \mathrm{mg}$ group passed from the surgical table to the stretcher. None of the $10 \mathrm{mg}$ patients passed without help from the surgical table to the stretcher (Table 2).

The low-dose group had significantly less motor block than the high-dose group (Table 3 and 4). All G10 patients had complete motor block at 10 minutes while none at G5. At 120 minutes no G5 patient had any degree of blockade in which G10 still had a block-motor grade 1 .

There was a significant difference in pain at different times between the group that did not receive any type of anesthesia when compared to patients receiving spinal anesthesia ( $\mathrm{p}$ $<0.05$ ) (Table 5).

In terms of satisfaction, there was a significant difference between the group that did not receive anesthesia when compared to the groups receiving spinal anesthesia $(p<0.05)$ (Table 6).

In our study we monitored intra-operative heart rate, noninvasive blood pressure, $\mathrm{SpO}_{2}$. There was no statistical difference in 3 groups. There were no symptoms of nausea, vomiting or pruritus in any of the patients. No patients had symptoms that were consistent with a transient neurological deficit and postdural puncture headache.

Table 1. Demographics dates.

\begin{tabular}{llll}
\hline & Group=GN & Group=GR5 & Group=GR10 \\
\hline Age $(\mathrm{yr})$ & $51.1 \pm 6.5$ & $52.0 \pm 11.2$ & $52.7 \pm 8.9$ \\
Weight $(\mathrm{kg})$ & $63.6 \pm 10.9$ & $62.4 \pm 14.2$ & $68.5 \pm 14.3$ \\
Height $(\mathrm{cm})$ & $157.3 \pm 4.8$ & $157.8 \pm 6.7$ & $160.1 \pm 4.3$ \\
ASA Physical & $1=5$ & $1=5$ & $1=6$ \\
state & $2=5$ & $1=5$ & $2=4$ \\
\hline
\end{tabular}

Table 2. Latency, dispersion block, duration of surgery, duration of sensitive block, passage from surgical table to transport stretcher.

\begin{tabular}{llll}
\hline Data & Group=GN & Group=GR5 & Group=GR10 \\
\hline Latency (min) & NE & $3.22 \pm 0.9$ & $3.15 \pm 1.2$ \\
Dispersion block (moda) & NE & T10 & T8 \\
Duration surgery (min) & $8.4 \pm 4.1$ & $8.7 \pm 1.05$ & $9.6 \pm 1.6$ \\
Duration sensitive block (min) & NE & $33.3 \pm 4.4$ & $68.0 \pm 8.8$ \\
Passage to transport stretcher & 10 & 10 & 0 \\
\hline
\end{tabular}

$\mathrm{NE}=$ not evaluated

Table 3. Assessment of motor block of the limbs with bupivacaine $5 \mathrm{mg}$.

\begin{tabular}{llll}
\hline Motor block & $\mathbf{1 0}$ min & $\mathbf{6 0}$ $\mathbf{~ i n}$ & $\mathbf{1 2 0} \mathbf{~ m i n}$ \\
\hline Grade 0 & 8 & 8 & 10 \\
Grade 1 & 0 & 2 & 0 \\
Grade 2 & 2 & 0 & 0 \\
Grade 3 & 0 & 0 & 0 \\
\hline
\end{tabular}

Table 4. Assessment of motor block of the limbs with bupivacaine $10 \mathrm{mg}$.

\begin{tabular}{llll}
\hline Motor block & $\mathbf{1 0} \mathbf{~ m i n}$ & $\mathbf{6 0} \mathbf{~ m i n}$ & $\mathbf{1 2 0} \mathbf{~ m i n}$ \\
\hline Grade 0 & 0 & 0 & 2 \\
Grade 1 & 0 & 3 & 8 \\
Grade 2 & 0 & 7 & 0 \\
Grade 3 & 10 & 0 & 0 \\
\hline
\end{tabular}

Table 5. Assessment of pain at different times.

\begin{tabular}{lllll}
\hline Time & Group=GN & Group=GR5 & Group=GR10 & P Value \\
\hline T1 & 7 & 0 & 1 & 0.03 \\
T2 & 5 & 0 & 0 & 0.04 \\
T3 & 1 & 0 & 0 & 0.1 \\
\hline
\end{tabular}

Table 6. Patient satisfaction.

\begin{tabular}{llll}
\hline & Group=GN & Group=GR5 & Group=GR10 \\
\hline Good & 2 & 10 & 4 \\
Satisfactory & 6 & 0 & 6 \\
Bad & 2 & 0 & 0 \\
\hline
\end{tabular}

\section{Discussion}

In this hospital there was no routine regarding the use of anesthetic techniques for BTCC, being performed in some cases with and without anesthesia. There is no protocol for conducting anesthesia for BTCC in the various oncological hospitals in Brazil. This study showed that spinal anesthesia for BTCC provided better pain control at all times studied with better patient satisfaction compared to patients who did not receive anesthesia.

The reasons for pain and discomfort are multi factorial. It is cumbersome for the patient to retain the applicators along with the vaginal packing for long duration. Bladder catheterization and skin sutures add to the pain. This discomfort is compounded by isolation of the patient, being confined to bed without any movement. The pain is more severe in case of transperineal template application as the needles penetrate through the skin right into the parametrium. The pain is considerably worsened by patient movement from operating table to the trolley, to the simulator and from there to the bed. In the ward close supervision is severely limited by the need to reduce radiation exposure to staff. All these factors make brachytherapy an unpleasant experience for the patients [6]. As demonstrated in the present study, since pain occurred in $70 \%$ of the patients in the group without anesthesia.

In 2006, a retrospective study evaluated the anesthesia experience for brachytherapy for five and a half years and presented regional anesthesia as the most used technique [3]. In 2007, the publication compared several anesthetic techniques in BTCC, concluding that general anesthesia was 
associated with complications that did not exist in the spinal anesthesia group, besides regional anesthesia to promote postoperative analgesia [7]. In our study, there was a significant reduction of pain when we compared spinal anesthesia with the group without anesthesia and the satisfaction was also greater in the group submitted to spinal anesthesia.

Various techniques of anesthesia have been described for intracavitory radiotherapy for cervical cancer. Each technique has its own advantage and some disadvantages also. In the present study we compared the different doses (10 mg with 5 $\mathrm{mg}$ ) of hyperbaric bupivacaine for spinal anesthesia for daystay brachytherapy for carcinoma of the cervix, with a view to shortening the time to full recovery, without compromising quality of analgesia during the procedure. The study showed a statistically significantly shorter time to readiness for hospital discharge in the low-dose group, with less motor block. Both groups had minimal side-effects.

In a retrospective analysis of records of 1,622 anesthetic procedures in 952 patients, they observed incidence of hypotension and bradycardia in about $10 \%$ patients with regional anaesthesia [3]. In another study the incidence of hypotension was 5.7\% under spinal anaesthesia [7]. Differently from this study [7], in our study with 10 and $5 \mathrm{mg}$ of $0.5 \%$ hyperbaric bupivacaine no case of hypotension was observed. Similarly found in another study [7], no case of bradycardia was observed in spinal anesthesia with both doses in our study. This low incidence of complication is probably because we kept level of block not higher than T8 with $10 \mathrm{mg}$ and $\mathrm{T} 10 \mathrm{com} 5 \mathrm{mg}$ of hyperbaric bupivacaine.

The incidence of headache after spinal anesthesia varies greatly between studies. Studying 5050 patients, 40 developed headaches post-spinal anesthesia, resulting in an incidence of $0.8 \%$ [8]. This work has shown that thinner needles provide low incidence of headache after spinal anesthesia, when with more calibrated needles of the same type. When pencil tip needles are used, they cause minor incidence of headache when compared to needles even cutting edge gauge. In this study with $27 \mathrm{G}$ gauge cutting point needle there was no report of headache after spinal anesthesia.

Hyperbaric bupivacaine was preferred to isobaric bupivacaine because of its more consistent and reliable subarachnoid spread, as well as its shorter duration of complete motor blockade [9]. Decreasing the dose of 10 to 5 $\mathrm{mg}$ of hyperbaric bupivacaine provided a lower degree of motor blockade and greater patient satisfaction without the occurrence of hypotension or bradycardia.

Cervical cancer is a disease feared by women because of the emotional significance the organ represents, since the uterus involves issues inherent to sexuality, femininity, and reproduction [10]. Brachytherapy can trigger considerable and challenging changes to the patient's physical and emotional well-being, from feeding, hygiene, sterility, sleep, rest and physiological elimination to work routine and social and family relationships [10]. We can observe that it also causes physical changes, which are responsible for increasing suffering and generating affective and emotional damages [10]. In this way, satisfaction assessment portrays the influence on the psychological and emotional aspects of BTCC in these patients.

\section{Conclusion}

Spinal anesthesia with low doses of hyperbaric bupivacaine is safe and necessary in BTCC, eliminating the pain phenomenon, which is frequent in patients who undergo treatment without anesthesia, since there is an inflammatory process in the circumference of the uterine cervix, making the region very sensitive. The patients' satisfaction was higher in the group submitted to spinal anesthesia with $5 \mathrm{mg}$ of hyperbaric bupivacaine $0.5 \%$ when compared to spinal anesthesia with $10 \mathrm{mg}$ and to the group without anesthesia. In conclusion, after this small study, spinal anesthesia became routine in this procedure and possibly to be implanted in all Brazilian Health System (SUS) units.

\section{References}

[1] INCA-Instituto Nacional de Câncer José de Alencar Gomes da Silva.

www2.inca.gov.br/wps/wcm/connect/tiposdecancer/site/home/ colo_utero/definição. Acesso em 15 janeiro 2018.

[2] INCA-Instituto Nacional de Câncer José de Alencar Gomes da Silva. Estimativa 2016: incidência de câncer no Brasil - Rio de Janeiro: INCA, 2015.

[3] Benrath J, Kozek-Langenecker S, Hüpfl M, Lierzy P, Gustorff B. Anaesthesia for brachytherapy $-5^{1 / 2} \mathrm{yr}$ of experience in 1622 procedures. Br J Anaesth, 2006; 96 (2): 195-200.

[4] Almeida LHRB, Pereira YBAS, Oliveira TA. Radiotherapy: perception of women with uterine-cervical cancer. Rev Bras Enferm, 2008; 61(4): 482-487.

[5] Dahl V, Raeder J. Regional anaesthesia in ambulatory surgery. Curr Opin Anaesthesiol, 2003; 16: 471-476.

[6] Smith MD, Todd JG, Symonds RP. Analgesia for pelvic brachytherapy. Br J Anaesthesiol 2002; 88: 270-276.

[7] R Jain, S Mishra, S Bhatnagar. Comparison of different anaesthetic techniques for high-dose-rate intracavitory. Radiotherapy for carcinoma cervix. The Internet Journal of Anesthesiology, 2007; 17 (1): 1-6.

[8] Imbelloni LE, Sobral MGC, Carneiro ANG. Postdural puncture headache and spinal needle design. Experience in 5050 Cases. Rev Bras Anestesiol, 2001; 51 (1): 43-52.

[9] Axelsson KH, Widman GB, Sundberg AE, Hallgren S. A double blind study of motor blockade in the lower limbs: studies during spinal anaesthesia with hyperbaric bupivacaine. Br J Anaesth, 1985; 57: 960-970.

[10] Lammering EAG, de Bock GH, Pras E, Reyners AKL, Mourits MJE. Sexual functioning of cervical cancer survivors: A review with a female perspective. Maturitas Eur Menopause J, 2012; 72 (4); 296-304. 